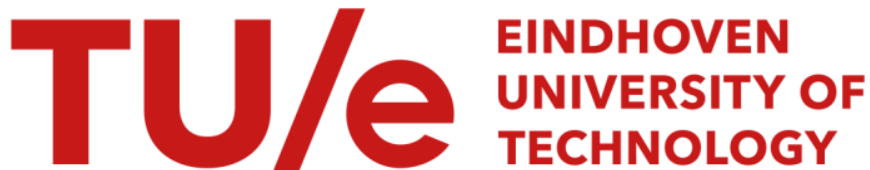

\section{Scale spaces on Lie groups}

\section{Citation for published version (APA):}

Duits, R., \& Burgeth, B. (2007). Scale spaces on Lie groups. In F. Sgallari, A. Murli, \& N. Paragios (Eds.), Proceedings of the First International Conference on Scale Space and Variational Methods in Computer Vision (SSVM 2007) 30 May - 2 June 2007, Ischia, Italy (pp. 300-312). (Lecture Notes in Computer Science; Vol. 4485). Springer. https://doi.org/10.1007/978-3-540-72823-8_26, https://doi.org/10.1007/978-3-540-72823-8

\section{DOI:}

10.1007/978-3-540-72823-8_26

10.1007/978-3-540-72823-8

\section{Document status and date:}

Published: 01/01/2007

\section{Document Version:}

Publisher's PDF, also known as Version of Record (includes final page, issue and volume numbers)

\section{Please check the document version of this publication:}

- A submitted manuscript is the version of the article upon submission and before peer-review. There can be important differences between the submitted version and the official published version of record. People interested in the research are advised to contact the author for the final version of the publication, or visit the $\mathrm{DOI}$ to the publisher's website.

- The final author version and the galley proof are versions of the publication after peer review.

- The final published version features the final layout of the paper including the volume, issue and page numbers.

Link to publication

\section{General rights}

Copyright and moral rights for the publications made accessible in the public portal are retained by the authors and/or other copyright owners and it is a condition of accessing publications that users recognise and abide by the legal requirements associated with these rights.

- Users may download and print one copy of any publication from the public portal for the purpose of private study or research.

- You may not further distribute the material or use it for any profit-making activity or commercial gain

- You may freely distribute the URL identifying the publication in the public portal.

If the publication is distributed under the terms of Article 25fa of the Dutch Copyright Act, indicated by the "Taverne" license above, please follow below link for the End User Agreement:

www.tue.nl/taverne

Take down policy

If you believe that this document breaches copyright please contact us at:

openaccess@tue.nl

providing details and we will investigate your claim. 


\title{
Scale Spaces on Lie Groups ${ }^{\star}$
}

\author{
Remco Duits and Bernhard Burgeth \\ Eindhoven University of Technology, Dept. of Biomedical Engineering and Dept. \\ Applied Mathematics and Computer Science, The Netherlands \\ R.Duits@tue.nl \\ Saarland University, Dept. of Mathematics and Computer Science, Germany \\ burgeth@mia.uni-saarland.de
}

\begin{abstract}
In the standard scale space approach one obtains a scale space representation $u: \mathbb{R}^{d} \rtimes \mathbb{R}^{+} \rightarrow \mathbb{R}$ of an image $f \in \mathbb{L}_{2}\left(\mathbb{R}^{d}\right)$ by means of an evolution equation on the additive group $\left(\mathbb{R}^{d},+\right)$. However, it is common to apply a wavelet transform (constructed via a representation $\mathcal{U}$ of a Lie-group $G$ and admissible wavelet $\psi$ ) to an image which provides a detailed overview of the group structure in an image. The result of such a wavelet transform provides a function $g \mapsto\left(\mathcal{U}_{g} \psi, f\right)_{\mathbb{L}_{2}\left(\mathbb{R}^{2}\right)}$ on a group $G$ (rather than $\left(\mathbb{R}^{d},+\right)$ ), which we call a score. Since the wavelet transform is unitary we have stable reconstruction by its adjoint. This allows us to link operators on images to operators on scores in a robust way. To ensure $\mathcal{U}$-invariance of the corresponding operator on the image the operator on the wavelet transform must be left-invariant. Therefore we focus on leftinvariant evolution equations (and their resolvents) on the Lie-group $G$ generated by a quadratic form $Q$ on left invariant vector fields. These evolution equations correspond to stochastic processes on $G$ and their solution is given by a group convolution with the corresponding Green's function, for which we present an explicit derivation in two particular image analysis applications. In this article we describe a general approach how the concept of scale space can be extended by replacing the additive group $\mathbb{R}^{d}$ by a Lie-group with more structure
\end{abstract}

\section{Introduction}

In the standard scale space approach one obtains a scale space representation $u: \mathbb{R}^{d} \rtimes \mathbb{R}^{+} \rightarrow \mathbb{R}$ of a square integrable image $f: \mathbb{R}^{d} \rightarrow \mathbb{R}$ by means of an evolution equation on the additive group $\left(\mathbb{R}^{d},+\right)$. It follows by the scale space axioms that the only allowable linear scale space representations are the so-called $\alpha$-scale space representations determined by the following linear system

$$
\left\{\begin{array}{l}
\partial_{s} u=-(-\Delta)^{\alpha} u, \quad 0<\alpha \leq 1 \\
u(\cdot, s) \in \mathbb{L}_{2}\left(\mathbb{R}^{d}\right) \text { for all } s>0 \text { and } u(\cdot, s) \rightarrow 0 \text { uniformly as } s \rightarrow \infty \\
u(\cdot, 0)=f
\end{array}\right.
$$

\footnotetext{
* The Dutch Organization for Scientific Research is gratefully acknowledged for financial support

1 This article provides the theory and general framework we applied in [9], [5], 8$]$.
} 
including both Gaussian $\alpha=1$ and Poisson scale space $\alpha=\frac{1}{2},[6]$. By the translation invariance axiom these scale space representations are obtained via a convolution on the additive group $\mathbb{R}^{d}$. For example if $d=2, \alpha=1$ the evolution system (1) is a diffusion system and the scale space representation is obtained by $u(\mathbf{x}, s)=\left(G_{s} * f\right)(\mathbf{x})$ where $G_{s}(\mathbf{x})=\frac{1}{4 \pi s} e^{-\frac{\|\mathbf{x}\|^{2}}{4 s}}$ denotes the Gaussian kernel. Its resolvent equation (obtained by Laplace transform with respect to scale) is

$$
(-\Delta+\gamma I) u=f \Leftrightarrow u=(-\Delta+\gamma I)^{-1} f
$$

the solution of which is given by $u(\mathbf{x}, \gamma)=\left(R_{\gamma} * f\right)(\mathbf{x})$, where the kernel

$$
R_{\gamma}(\mathbf{x})=-\frac{1}{2 \pi \gamma^{2}} k_{0}\left(\gamma^{-1}\|\mathbf{x}\|\right), \quad \mathbf{x} \in \mathbb{R}^{2},
$$

equals the Laplace transform of the Gaussian kernel $s \mapsto G_{s}(\mathbf{x})$ expressed in the well-known BesselK-function $k_{0}$. To this end we note that $\int_{0}^{\infty} e^{s \Delta} f e^{-\gamma s} \mathrm{~d} s=$ $-\gamma(\Delta-\gamma I)^{-1} f$. Although this explicit convolution kernel is not common in image analysis it plays an important mostly implicit role as it occurs in the minimization of a first order Sobolev norm

$$
\mathcal{E}(u)=\|u\|_{\mathbb{H}^{1}\left(\mathbb{R}^{d}\right)}^{2}=\|u-f\|_{\mathbf{L}_{2}\left(\mathbb{R}^{2}\right)}^{2}+\|\nabla u\|_{\mathbf{L}_{2}\left(\mathbb{R}^{2}\right)}^{2} .
$$

Indeed by some elementary variational calculus and partial integration one gets $\mathcal{E}^{\prime}(u) v=((\gamma I-\Delta) u-\gamma f, v)$ and $\mathcal{E}^{\prime}(u) v=0$ for all $v \in \mathbb{L}_{2}\left(\mathbb{R}^{2}\right)$ iff $u=\gamma(\gamma I-\Delta)^{-1} f$, where $R_{\gamma}=\gamma(\gamma I-\Delta)^{-1} \delta=\gamma \int_{0}^{\infty} e^{-\gamma s} e^{s \Delta} \delta \mathrm{d} s=\gamma \int_{0}^{\infty} e^{-\gamma s} G_{s}$ d $s$. The connection between a linear scale space and its resolvent equation is also relevant for stochastic interpretation. Consider $f$ as a probability density distribution of photons. Then its scale space representation evaluated at a point $(\mathbf{x}, s)$ in scale space, $u(\mathbf{x}, s)$, corresponds to the probability density of finding a random walker in a Wiener process at position $\mathbf{x}$ at time/scale $s>0$. In such a process traveling time is negatively exponentially distributed.Now the probability density of finding a random walker at position $\mathbf{b}$ given the initial distribution $f$ equals

$$
p(\mathbf{b})=\int_{0}^{\infty} p(\mathbf{b} \mid T=t) p(T=t) \mathrm{d} t=\gamma \int_{0}^{\infty} e^{-\gamma t}\left(G_{t} * f\right)=\gamma(\gamma I-\Delta)^{-1} f .
$$

In the remainder of this article we are going to repeat the above results for other Lie-groups than $\left(\mathbb{R}^{d},+\right)$. Just like ordinary convolutions on $\mathbb{R}^{d}$ are the only translation invariant kernel operators, it is easy to show that the only left invariant operators on a Lie-group $G$ are $G$-convolutions, which are given by

$$
\left(K *_{G} f\right)(g)=\int_{G} K\left(h^{-1} g\right) f(h) \mathrm{d} \mu_{G}(g),
$$

where $\mu_{G}$ is the left invariant haar measure of the group $G$. However, if the Lie group $G$ is not commutative it is challenging to compute the analogues of the Gaussian and corresponding resolvent kernel. 
Definition 1. Let $H$ be a Hilbert space and let $G$ be a group with unit element e. Let $\mathcal{B}(H)$ denote the space of bounded operators on $H$. Then a mapping $\mathcal{R}: G \rightarrow$ $\mathcal{B}(H)$ given by $g \mapsto \mathcal{R}_{g}$, where $\mathcal{R}_{g}$ is bounded for all $g \in G$, is a representation if $\mathcal{R}_{g} \mathcal{R}_{h}=\mathcal{R}_{\text {gh }}$ for all $g, h \in G$, with $\mathcal{R}_{e}=I$. If for every $g \in G$ the operator $\mathcal{R}_{g}$ is unitary (so $\left\|\mathcal{R}_{g} f\right\|=\|f\|$ for all $f \in H$ ) the representation is called unitary.

Definition 2. An operator on $\mathbb{L}_{2}(G)$ is left invariant if it commutes with the left regular representation given by $\left(\mathcal{L}_{g} U\right)(h)=U\left(g^{-1} h\right), \quad U \in \mathbb{L}_{2}(G), h, g \in G$. So an operator $\Phi$ on $\mathbb{L}_{2}(G)$ is left invariant if $\mathcal{L}_{g} \circ \Phi=\Phi \circ \mathcal{L}_{g}$ for all $g \in G$.

The motivation for our generalization of scale space theory to arbitrary Liegroups comes from wavelet-theory applications where one applies a wavelet transform $\mathcal{W}_{\psi}: \mathbb{L}_{2}\left(\mathbb{R}^{d}\right) \rightarrow \mathbb{L}_{2}(G)$ to the original image $f \in \mathbb{L}_{2}\left(\mathbb{R}^{d}\right)$ to provide more insight in the group structure of an image. Such a transform is usually given by

$$
\left(\mathcal{W}_{\psi} f\right)(g)=\left(\mathcal{U}_{g} \psi, f\right)_{\mathbb{L}_{2}\left(\mathbb{R}^{d}\right)}
$$

where the wavelet $\psi$ is admissible 2 and a unitary representation $\mathcal{U}: G \rightarrow$ $\mathcal{B}\left(\mathbb{L}_{2}\left(\mathbb{R}^{d}\right)\right)$ of a certain Lie-group $G$. Provided that $\psi$ is admissible such wavelet transform is an isometry from $\mathbb{L}_{2}\left(\mathbb{R}^{d}\right)$ into $\mathbb{L}_{2}(G)$ and thereby we have perfectly stable reconstruction by the adjoint wavelet transformation; $f=\mathcal{W}_{\psi}^{*}\left(\mathcal{W}_{\psi} f\right)$, allowing us to link scale operators on images and their scores $U_{f}:=\mathcal{W}_{\psi} f$ in a stable manner. We must consider left invariant operators on $U_{f}$, see figure 1

\section{Scale Spaces on Lie-Groups: The General Recipe}

In this section we provide a general recipe for scale spaces on Lie-groups. In contrast to other work on scale spaces via Lie-groups we consider the left-invariant vector fields on the Lie-group itself, rather than infinitesimal generators 3 of the representation $\mathcal{U}$ on $\mathbb{L}_{2}\left(\mathbb{R}^{d}\right)$, [15]. The main advantage over the infinitesimal generators is that these vector fields are defined on the group manifold $G$ rather than $\mathbb{R}^{d}$. Moreover, they give rise to left-invariant evolution equations on $\mathbb{L}_{2}(G)$.

First we compute the left-invariant vector-fields on a Lie-group. By definition those are vector fields on $G$, with unit element $e$, such that

$$
X_{g} f=X_{e}\left(f \circ L_{g}\right)
$$

for all $f \in C^{\infty}\left(\Omega_{g}\right)$ defined on some open neighborhood $\Omega_{g}$ of $g \in G$, where we note that $L_{g} h=g h$ denotes the left multiplication on the Lie-group. Note that by (6) the restriction $X_{g}$ of a left invariant vector field $X$ is always connected by its restriction to the unity element $X_{e}$. Consequently such left invariant vector

\footnotetext{
${ }^{2}$ this is a condition on $\psi$ to ensure that $\left\|\mathcal{W}_{\psi} f\right\|_{\mathbb{L}_{2}(G)}=\|f\|_{\mathbb{L}_{2}\left(\mathbb{R}^{d}\right)}$ for all $f \in \mathbb{L}_{2}\left(\mathbb{R}^{d}\right)$.

${ }^{3}$ The left invariant vector fields are obtained via the Lie-algebra $T_{e}(G)=\left\{A_{1}, \ldots A_{n}\right\}$ by means of the derivative of the right-regular representation on $\mathbb{L}_{2}(G)$ by $\left\{\mathrm{d} \mathcal{R}\left(A_{i}\right)\right\}_{i=1}^{n}$, whereas the infinitesimal generators are obtained by the derivative of some representation $\mathcal{U}$ on $\mathbb{L}_{2}\left(\mathbb{R}^{d}\right)$ by $\left\{\mathrm{d} \mathcal{U}\left(A_{i}\right)\right\}_{i=1}^{n}$.
} 


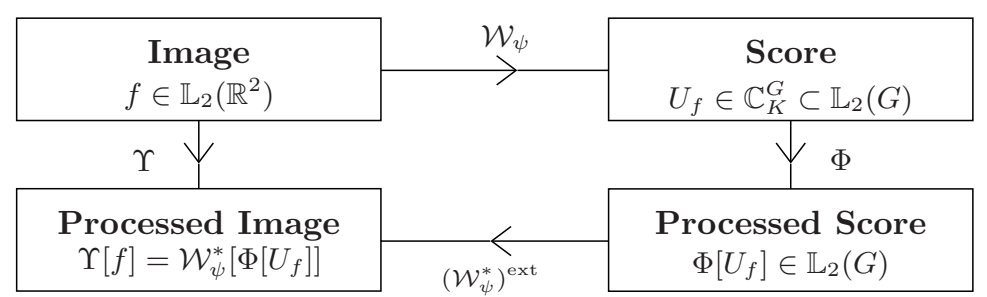

Fig. 1. The complete scheme; for admissible vectors $\psi$ the linear map $\mathcal{W}_{\psi}$ is unitary from $\mathbb{L}_{2}\left(\mathbb{R}^{2}\right)$ onto a closed subspace $V$ of $\mathbb{L}_{2}(G)$. So we can uniquely link a transformation $\Phi: V \rightarrow V$ on the wavelet domain to a transformation in the image domain $\Upsilon=\left(\mathcal{W}_{\psi}^{*}\right)^{\text {ext }} \circ \Phi \circ \mathcal{W}_{\psi} \in \mathcal{B}\left(\mathbb{L}_{2}\left(\mathbb{R}^{d}\right)\right)$, where $\left(\mathcal{W}_{\psi}^{*}\right)^{\text {ext }}$ is the extension of the adjoint to $\mathbb{L}_{2}(G)$ given by $\left(\mathcal{W}_{\psi}^{*}\right)^{e x t} U=\int_{G} \mathcal{U}_{g} \psi U(g) \mathrm{d} \mu_{G}(g), U \in \mathbb{L}_{2}(G)$. It is easily verified that $\mathcal{W}_{\psi} \circ \mathcal{U}_{g}=\mathcal{L}_{g} \circ \mathcal{W}_{\psi}$ for all $g \in G$. As a result the net operator on the image domain $\Upsilon$ is invariant under $\mathcal{U}$ (which is highly desirable) if and only if the operator in the wavelet domain is left invariant, i.e. $\Upsilon \circ \mathcal{U}_{g}=\mathcal{U}_{g} \circ \Upsilon$ for all $g \in G$ if and only if $\Phi \circ \mathcal{L}_{g}=\mathcal{L}_{g} \circ \Phi$ for all $g \in G$. For more details see [4Thm. 21 p.153. In our applications [8], [5], 4] we usually take $\Phi$ as a concatenation of non-linear invertible grey-value transformations and linear left invariant (anisotropic) scale space operators, for example $\Phi\left(U_{f}\right)=\gamma^{2 / p}\left((Q(\underline{A})-\gamma I)^{-1}\left(U_{f}\right)^{p}(Q(\underline{A})-\gamma I)^{-1}\left(U_{f}\right)^{p}\right)^{1 / p}$, for some sign-preserving power with exponent $p>0$. A nice alternative, however, are non-linear adaptive scale spaces on Lie-groups as explored for the special case $G=\mathbb{R}^{2} \rtimes \mathbb{T}$ in 9 .

fields are isomorphic to the tangent space $T_{e}(G)$ at the unity element $e \in G$, also known as the Lie-Algebra of $G$. The isomorphism between $T_{e}(G)$ and the space of left invariant vector fields $\mathcal{L}(G)$ on $G$ (considered as differential operators) is

$$
T_{e}(G) \ni A \leftrightarrow \mathcal{A} \in \mathcal{L}(G) \Leftrightarrow \mathcal{A}_{g} \phi=A(h \mapsto \phi(g h)) \text {, for all } \phi \in C^{\infty}\left(\Omega_{g}\right) .
$$

The Lie-product on $T_{e}(G)$ is given by $[A, B]=\lim _{t \downarrow 0} \frac{a(t) b(t)(a(t))^{-1}(b(t))^{-1}-e}{t^{2}}$, where $t \mapsto a(t)$ resp. $t \mapsto b(t)$ are any smooth curves in $G$ with $a(0)=b(0)=e$ and $a^{\prime}(0)=A$ and $b^{\prime}(0)=B$, whereas the Lie-product on $\mathcal{L}(G)$ is given by $[\mathcal{A}, \mathcal{B}]=\mathcal{A B}-\mathcal{B} \mathcal{A}$. The mapping (7) is an isomorphism between $T_{e}(G)$ and $\mathcal{L}(G)$, so $A \leftrightarrow \mathcal{A}$ and $B \leftrightarrow \mathcal{B}$ imply $[A, B] \leftrightarrow[\mathcal{A}, \mathcal{B}]$.

Consider a Lie-group $G$ of finite dimension, with Lie-algebra $T_{e}(G)$. Let $\left\{A_{1}, \ldots, A_{n}\right\}$ be a basis within this Lie-algebra. Then we would like to construct the corresponding left invariant vector fields $\left\{\mathcal{A}_{1}, \ldots, \mathcal{A}_{n}\right\}$ in a direct way. This is done by computing the derivative $\mathrm{d} \mathcal{R}$ of the right-regular representation $\mathcal{R}: G \mapsto \mathcal{B}\left(\mathbb{L}_{2}(G)\right)$. The right regular representation $\mathcal{R}: G \rightarrow \mathcal{B}\left(\mathbb{L}_{2}(G)\right)$ is given by $\left(\mathcal{R}_{g} \Phi\right)(h)=\Phi(h g)$, for all $\Phi \in \mathbb{L}_{2}(G)$ and almost every $h \in G$. It is left-invariant and its derivative $\mathrm{d} \mathcal{R}$, which maps $T_{e}(G)$ onto $\mathcal{L}(G)$, is given by

$$
(\mathrm{d} \mathcal{R}(A) \Phi)(g)=\lim _{t \rightarrow 0} \frac{\left(\mathcal{R}_{\exp (t A)} \Phi\right)(g)-\Phi(g)}{t}, A \in T_{e}(G), \Phi \in \mathbb{L}_{2}(G), g \in G .
$$

So a basis for $\mathcal{L}(G)$ is given by

$$
\left\{\mathcal{A}_{1}, \mathcal{A}_{2}, \ldots, \mathcal{A}_{n}\right\}:=\left\{\mathrm{d} \mathcal{R}\left(A_{1}\right), \mathrm{d} \mathcal{R}\left(A_{2}\right), \ldots, \mathrm{d} \mathcal{R}\left(A_{n}\right)\right\} .
$$


Now let $Q=Q^{\mathbf{D}, \mathbf{a}}$ be some bilinear/quadratic form on $\mathcal{L}(G)$, i.e.

$$
Q^{\mathbf{D}, \mathbf{a}}\left(\mathcal{A}_{1}, \mathcal{A}_{2}, \ldots, \mathcal{A}_{n}\right)=\sum_{i=1}^{n} a_{i} \mathcal{A}_{i}+\sum_{j=1}^{n} D_{i j} \mathcal{A}_{i} \mathcal{A}_{j}, \quad a_{i}, D_{i j} \in \mathbb{R}
$$

where we will assume that the matrix $D=\left[D_{i j}\right]$ is symmetric and positive semi-definite, and consider the following evolution equations

$$
\left\{\begin{array}{l}
\partial_{s} W=Q\left(\mathcal{A}_{1}, \mathcal{A}_{2}, \ldots, \mathcal{A}_{n}\right) W \\
\lim _{s \downarrow 0} W(\cdot, s)=U_{f}(\cdot)
\end{array}\right.
$$

the solutions of which we call the $G$-scale space representation of initial condition $U_{f}$ (which is the score obtained from image $f$ by $\mathcal{W}_{\psi}[f]$ ). The corresponding $G$ Tikhonov regularization due to minimization of

$$
\mathcal{E}(u)=\|u\|_{\mathbb{H}^{1}(G)}^{2}=\|u-f\|_{\mathbf{L}_{2}(G)}^{2}+\sum_{i=1}^{d} D_{i i}\left\|\mathcal{A}_{i} u\right\|_{\mathbf{L}_{2}(G)}^{2} \text { is again obtained by }
$$

Laplace transform with respect to scale yielding the following resolvent equations

$$
\left(-Q\left(\mathcal{A}_{1}, \mathcal{A}_{2}, \ldots, \mathcal{A}_{n}\right)+\gamma I\right) P_{\gamma}=\gamma U_{f},
$$

with $P_{\gamma}=\gamma \mathcal{L}(s \mapsto W(\cdot, s))(\gamma)$ and where traveling timeof a random walker in $G$ is assumed to be negatively exponentially distributed with $s \sim \operatorname{NE}(\gamma)$.

We distinguish between two types of scale space representations, the cases where $Q$ is non-degenerate and the cases where $Q$ is degenerate. If $Q$ is nondegenerate the principal directions in the diffusion span the whole tangent space in which case it follows that (11) gives rise to a strongly continuous semi-group, generated by a hypo-elliptic operator $A$, [12, such that the left-invariant operators $U_{f} \mapsto W(\cdot, s)$ and $U_{f} \mapsto P_{\gamma}$ are bounded operators on $\mathbb{L}_{\infty}(G)$ for all $\gamma, s>0$ and by means of the Dunford-Pettis theorem 2] if follows that the solutions of (11) and (12) are given by $G$-convolutions with the corresponding smooth Green's functions

$$
\begin{aligned}
& W(g, s)=\left(K_{s} *_{G} U_{f}\right)(g), \quad K_{s} \in C^{\infty}(G), s>0 \\
& P_{\gamma}(g)=\left(R_{\gamma} *_{G} U_{f}\right)(g), \quad R_{\gamma} \in C^{\infty}(G \backslash\{e\}),
\end{aligned}
$$

where $K_{s}$ and $R_{\gamma}$ are connected by Laplace transform $R_{\gamma}=\gamma \mathcal{L}\left(s \mapsto K_{s}\right)(\gamma)$.

The most interesting cases, however, arise if $Q$ is degenerate. If $Q$ is degenerate it follows by the general result by [11] the solutions of (11) and (13) are still given by group convolutions (13). The question though is whether the convolution kernels are to be considered in distributional sense only or if they are smooth functions. If $D=0$ the convolution kernels are highly singular and concentrated at the exponential curves within the Lie-group. If $D \neq 0$ is degenerate diffusion takes place only in certain direction(s) and we can write $Q(\underline{\mathcal{A}})=\sum_{j=1}^{d} \tilde{\mathcal{A}}_{j}^{2}+$ $\tilde{\mathcal{A}}_{0}, \quad d=\operatorname{rank}(Q)$ where $\mathcal{A}_{0}$ is the convection part of $Q(\underline{\mathcal{A}})$ and where $\tilde{\mathcal{A}}_{j}$ are $d$-independent directions along which $Q$ is not degenerate. Now it is the question 
whether the non-commutativity of the vector fields results in a smoothing along the other directions. By employing the results of Hörmander [12] and Hebisch [11] we obtain the following necessary and sufficient conditions for smooth scale space representations of the type (13): Among the vector fields

$$
\tilde{\mathcal{A}}_{j_{1}},\left[\tilde{\mathcal{A}}_{j_{1}}, \tilde{\mathcal{A}}_{j_{2}}\right], \ldots\left[\tilde{\mathcal{A}}_{j_{1}},\left[\tilde{\mathcal{A}}_{j_{2}},\left[\tilde{\mathcal{A}}_{j_{3}}, \ldots, \tilde{\mathcal{A}}_{j_{k}}\right]\right]\right] \ldots, j_{i}=0,1, \ldots d
$$

there exist $n$ which are linearly independent.

\section{$3 \quad$ Examples}

\section{Spatial-Frequency Enhancement via left invariant scale spaces on Gabor Transforms: $G=H_{3}, Q(\underline{\mathcal{A}})=D_{11}\left(\mathcal{A}_{1}\right)^{2}+D_{22}\left(\mathcal{A}_{2}\right)^{2}$}

Consider the Heisenberg group $H_{2 d+1}=\mathbb{C}^{d} \ltimes \mathbb{R}$ with group product: $g g^{\prime}=(\mathbf{z}, t)\left(\mathbf{z}^{\prime}, t^{\prime}\right)=\left(\mathbf{z}+\mathbf{z}^{\prime}, t+t^{\prime}+2 \operatorname{Im}\left\{\sum_{j=1}^{d} z_{j} \overline{z_{j}^{\prime}}\right\}\right), \quad$ where $z_{j}=x_{j}+i \omega_{j} \in \mathbb{C}$ and consider its representations $U_{(\mathbf{x}, \boldsymbol{\omega}, t)}^{\lambda}$ on $\mathbb{L}_{2}\left(\mathbb{R}^{d}\right)$ :

$$
\left(\mathcal{U}_{(\mathbf{z}, t)}^{\lambda} \psi\right)(\boldsymbol{\xi})=e^{i \lambda\left((\boldsymbol{\xi}, \boldsymbol{\omega})+\frac{t}{4}-\frac{1}{2}(\mathbf{x}, \boldsymbol{\omega})\right)} \psi(\boldsymbol{\xi}-\mathbf{x}), \quad \psi \in \mathbb{L}_{2}\left(\mathbb{R}^{d}\right), \mathbf{x}, \boldsymbol{\omega} \in \mathbb{R}^{d}, \lambda \in \mathbb{R}
$$

The corresponding wavelet transform is the windowed Fourier/Gabor transform:

$$
\left(\mathcal{W}_{\psi}[f]\right)(g)=\left(\mathcal{U}_{g} \psi, f\right)_{\mathbb{L}_{2}\left(\mathbb{R}^{d}\right)}=e^{-i \lambda\left(\frac{t}{4}-\frac{(\mathbf{x}, \boldsymbol{\omega})}{2}\right)} \int_{\mathbb{R}^{d}} \overline{\psi(\boldsymbol{\xi}-\mathbf{x})} f(\boldsymbol{\xi}) e^{-i \lambda(\boldsymbol{\xi}, \boldsymbol{\omega})} \mathrm{d} \boldsymbol{\xi}
$$

This is useful in practice as it provides a score of localized frequencies in signal $f$. Denote the phase subgroup of $H_{2 d+1}$ by $\Theta=\{(\mathbf{0}, \mathbf{0}, t) \mid t \in \mathbb{R}\}$.

Now $\mathcal{U}^{\lambda}$ is a unitary, irreducible and square integrable representation with respect to $H_{2 d+1} / \Theta$ with invariant measure $\mathrm{d} \mu_{H_{2 d+1} / \Theta}(g)=\mathrm{d} \boldsymbol{\omega} \mathrm{d} \mathbf{x}$. Therefore by the theory of vector coherent states, [1, we employ that 4

$$
\int_{\mathbb{R}^{d}} \int_{\mathbb{R}^{d}}\left|\mathcal{W}_{\psi}[f](\mathbf{x}, \boldsymbol{\omega}, 0)\right|^{2} \mathrm{~d} \mathbf{x} \mathrm{d} \boldsymbol{\omega}=C_{\psi} \int_{\mathbb{R}^{d}}|f(\mathbf{x})|^{2} \mathrm{~d} \mathbf{x},
$$

for all $f \in \mathbb{L}_{2}\left(\mathbb{R}^{d}\right)$ and for all $\psi \in \mathbb{L}_{2}\left(\mathbb{R}^{d}\right)$. As a result we obtain a perfectly stable reconstruction by means of the adjoint wavelet transform

$$
\begin{aligned}
& f=\frac{1}{C_{\psi}} \mathcal{W}_{\psi}^{*} \mathcal{W}_{\psi} f=\frac{1}{C_{\psi}} \int_{H_{2 d+1} / \Theta} \mathcal{W}_{\psi}[f](g) \mathcal{U}_{g} \psi \mathrm{d} \mu_{H_{2 d+1} / \Theta}(g), \text { i.e. } \\
& f(\boldsymbol{\xi})=\frac{1}{C_{\psi}} \int_{\mathbb{R}^{d}} \int_{\mathbb{R}^{d}}\left(\mathcal{W}_{\psi} f\right)(\mathbf{x}, \boldsymbol{\omega}, t) e^{i \lambda[(\boldsymbol{\xi}, \boldsymbol{\omega})+(t / 4)-(1 / 2)(\mathbf{x}, \boldsymbol{\omega})]} \psi(\boldsymbol{\xi}-\mathbf{x}) \mathrm{d} \mathbf{x} \mathrm{d} \boldsymbol{\omega},
\end{aligned}
$$

for almost every $\boldsymbol{\xi} \in \mathbb{R}^{d}$ and all $f \in \mathbb{L}_{2}\left(\mathbb{R}^{d}\right)$.

Now that a stable connection between an image $f$ and its Gabor-transform $\mathcal{W}_{\psi}[f]$ is set we can think of left invariant scale spaces on the space of Gabor transforms which is embedded in $\mathbb{L}_{2}\left(H_{2 d+1} / \Theta\right)$. Following the general recipe

${ }^{4}$ Note that $C_{\psi}=\int_{\mathbb{R}^{d}} \int_{\mathbb{R}^{d}}\left|\left(\mathcal{U}_{(\mathbf{x}+i \boldsymbol{\omega}, 0)}^{\lambda} \psi, \psi\right)\right|^{2} \mathrm{~d} \mathbf{x} \mathrm{d} \boldsymbol{\omega}=\frac{(2 \pi)^{d}}{\lambda}\|\psi\|_{\mathbb{L}_{2}\left(\mathbb{R}^{d}\right)}^{4}<\infty$ for all $\psi \in \mathbb{L}_{2}\left(\mathbb{R}^{2}\right)$. 
as described in Section 2 we compute the left invariant vector fields from the $2 d+1$-dimensional Lie-algebra $T_{e}(G)$ spanned by

$T_{e}(G)=\left\langle\left\{\left(\mathbf{e}_{i}, \mathbf{0}, 0\right),\left(\mathbf{0}, \mathbf{e}_{j}, 0\right),(\mathbf{0}, \mathbf{0}, 1)\right\}_{i, j=1, \ldots d}\right\rangle=\left\langle\left\{A_{1}, \ldots, A_{d}, A_{d+1}, \ldots, A_{2 d}, A_{2 d+1}\right\}\right\rangle$

by means of $\mathcal{A}_{i} \psi=\mathrm{d} \mathcal{R}\left(A_{i}\right) \psi=\lim _{t \rightarrow 0} \frac{1}{t}\left(R\left(e^{t A_{i}}\right)-I\right) \psi$. A straightforward calculation yields the following basis for $\mathcal{L}\left(H_{2 d+1}\right)$ :

$$
\mathcal{A}_{i}=\partial_{x_{i}}+2 \omega_{i} \partial_{t}, \mathcal{A}_{d+i}=\partial_{\omega_{i}}-2 x_{i} \partial_{t}, \text { for } i=1, \ldots, d \text {, and } \mathcal{A}_{2 d+1}=\partial_{t},
$$

the commutators of which are given by

$$
\left[\mathcal{A}_{i}, \mathcal{A}_{j}\right]=-4 \delta_{j, i+d} \mathcal{A}_{2 d+1}, i, j=1, \ldots, 2 d,\left[\mathcal{A}_{2 d+1}, \mathcal{A}_{j}\right]=0, j=1, \ldots, 2 d+1 .
$$

Here we only consider (11) for the case where the quadratic form equals

$$
Q(\underline{A})=\sum_{j=1}^{d} D_{j j}\left(\mathcal{A}_{j}\right)^{2}+D_{d+j, d+j}\left(\mathcal{A}_{d+j}\right)^{2} .
$$

Condition (14) is satisfied. In this case the scale space solutions $K_{s}^{\mathbf{D}}, P_{\gamma}^{\mathbf{D}}$ (13) initial condition $W(\cdot, \cdot, \cdot, 0)=U_{f} \in \mathbb{L}_{2}\left(H_{2 d+1}\right)$ of (11) are group convolutions (44) with the corresponding Green's functions $K_{s}^{\mathbf{D}}$ and $R_{\gamma}$. For $d=1$ we get:

$$
\begin{aligned}
& W^{\mathbf{D}}(x, \omega, t, s)=\left(K_{s}^{\mathbf{D}} *_{H_{3}} U_{f}\right)(x, \omega, t) \\
& =\iint_{\mathbb{R}} \int_{\mathbb{R}} K_{s}^{\mathbf{D}}\left(x-x^{\prime}, \omega-\omega^{\prime}, t-t^{\prime}-2\left(x \omega^{\prime}-x^{\prime} \omega\right)\right) U_{f}\left(x^{\prime}, \omega^{\prime}, t^{\prime}\right) \mathrm{d} t \mathrm{~d} \omega^{\prime} \mathrm{d} x^{\prime} \\
& P_{\gamma}^{\mathbf{D}}(x, \omega, t)=\left(R_{\gamma}^{\mathbf{D}} *_{H_{3}} U_{f}\right)(x, \omega, t) \\
& =\int_{\mathbb{R}} \int_{\mathbb{R}} \int_{\mathbb{R}^{+}} R_{\gamma}^{\mathbf{D}}\left(x-x^{\prime}, \omega-\omega^{\prime}, t-t^{\prime}-2\left(x \omega^{\prime}-x^{\prime} \omega\right)\right) U_{f}\left(x^{\prime}, \omega^{\prime}, t^{\prime}\right) \mathrm{d} t^{\prime} \mathrm{d} \omega^{\prime} \mathrm{d} x^{\prime} .
\end{aligned}
$$

Next we derive the Green's functions $K_{s}^{\mathbf{D}}$ and $R_{\gamma}$. First we note that in case $D_{j j}=D_{d+j, d+j}=\frac{1}{2}$ operator (16) coincides with Kohn's Laplacian, the fundamental solution of which is well-known [10]. As there exist several contradicting formulas for this Green's function, we summarize (for $d=1$ ) the correct derivation by Gaveau [10] which, together with the work of Lévy [13, provides important insight in the non-commutativity and the underlying stochastic process.

For $d=1$ the kernel $K_{s}^{\mathbf{D}}$ can be obtained by the Kohn Green's function $K_{s}:=K_{s}^{D_{11}=\frac{1}{2}, D_{22}=\frac{1}{2}}$ by means of a simple rescaling

$$
\begin{aligned}
& K_{s}^{\mathbf{D}}(x, y, t)=K_{s}\left(\sqrt{\frac{2}{D_{11}}} x, \sqrt{\frac{2}{D_{22}}} y, \frac{2}{\sqrt{D_{11} D_{22}}} t\right) \\
& K_{s}(x, \omega, t)=s^{-2} K_{1}\left(\frac{x}{\sqrt{s}}, \frac{\omega}{\sqrt{s}}, \frac{t}{s}\right) .
\end{aligned}
$$

Next we rewrite Kohn's $d$-dimensional Laplacian $\Delta_{K}$ in its fundamental form:

$$
\begin{aligned}
\Delta_{K} & =\sum_{i=1}^{d}\left(\partial_{x_{i}}\right)^{2}+\left(\partial_{\omega_{i}}\right)^{2}+4 \omega_{i} \partial_{x_{i}} \partial_{t}-4 x_{i} \partial_{\omega_{i}} \partial_{t}+4\left|z_{i}\right|^{2}\left(\partial_{t}\right)^{2} \\
& =\sum_{i=1}^{2 d+1} \sum_{j=1}^{2 d+1} \mathcal{A}_{i} g^{i j} \mathcal{A}_{j}=\sum_{i=1}^{2 d+1} \sum_{j=1}^{2 d+1} \mathcal{A}_{i}\left(\sigma^{T} \sigma\right)^{i j} \mathcal{A}_{j}=\sum_{k=1}^{2 d}\left(\sum_{j=1}^{2 d+1} \sigma^{k j} \mathcal{A}_{j}\right)^{2}
\end{aligned}
$$


with $G=\sigma^{T} \sigma \in \mathbb{R}^{(2 d+1) \times(2 d+1)}, G=\left[g^{i j}\right], \sigma=\left[\sigma^{k j}\right], \sigma \in \mathbb{R}^{2 d \times(2 d+1)}$ given by

$$
\sigma^{i j}=\left\{\begin{array}{cl}
\delta^{i j} & \text { if } i \leq 2 d, j \leq 2 d \\
2 w_{p} & \text { if } i=2 p-1 \text { and } j=2 d+1, p=1, \ldots, d \\
-2 x_{p} & \text { if } i=2 p \text { and } j=2 d+1, p=1, \ldots, d
\end{array}\right.
$$

where we recall that $z_{j}=x_{j}+i \omega_{j}$. By (19) the diffusion increments satisfy

$$
\left(d x^{1}, \ldots, d x^{d}, d \omega^{1}, \ldots, d \omega^{d}, d t\right)=\left(d x^{1}, \ldots, d x^{d}, d \omega^{1}, \ldots, d \omega^{d}\right) \sigma,
$$

so that $\mathrm{d} t=2 \sum_{j=1}^{d} \omega_{j} \mathrm{~d} x_{j}-x_{j} \mathrm{~d} \omega_{j}$. So in case $G=H_{2 d+1}$ the diffusion system (11) is the stochastic differential equation of the following stochastic process

$$
\left\{\begin{array}{l}
\mathbf{Z}(s)=\mathbf{X}(s)+i \mathbf{W}(s)=\mathbf{Z}_{0}+\boldsymbol{\xi} \sqrt{s}, \boldsymbol{\xi}=\left(\xi_{1}, \ldots, \xi_{d}\right), \xi_{j} \sim \mathcal{N}(0,1) \\
T(s)=2 \sum_{j=1}^{d} \int_{0}^{s} W_{j} \mathrm{~d} X_{j}-X_{j} \mathrm{~d} W_{j}, s>0
\end{array}\right.
$$

so the random variable $\mathbf{Z}=\left(Z_{1}, \ldots, Z_{d}\right)$ consists of $d$-independent Brownian motions in the complex plane The random variable $T(s)$ measures the deviation from a sample path with respect to a straight path $\mathbf{Z}(s)=\mathbf{Z}_{0}+s\left(\mathbf{Z}(s)-\mathbf{Z}_{0}\right)$ by means of the stochastic integral $T(s)=2 \sum_{j=1}^{d} \int_{0}^{s} W_{j} \mathrm{~d} X_{j}-X_{j} \mathrm{~d} W_{j}$.

To this end we note that for $5 \mapsto(x(s), \omega(s)) \in C^{\infty}\left(\mathbb{R}^{+}, \mathbb{R}^{2}\right)$ such that the straight-line from $X_{0}$ to $X(s)$ followed by the inverse path encloses an oriented surface $\Omega \in \mathbb{R}^{2}$, we have by Stokes' theorem that

$$
2 \mu(\Omega)=-\int_{0}^{s}\left(-X^{\prime}(t) W(t)+X(t) W^{\prime}(t)\right) \mathrm{d} t+0=\int_{0}^{s} W \mathrm{~d} X-X \mathrm{~d} W .
$$

Now we compute the Fourier transform $\mathcal{F}_{3} K_{1}$ of $K_{1}$ (with respect to $\left.(x, \omega, t)\right)$ :

$$
\begin{aligned}
\left(\mathcal{F}_{3} K_{1}\right)(\xi, \eta, \tau) & =\frac{1}{(2 \pi)^{\frac{3}{2}}} \int_{\mathbb{R}^{3}} e^{-i(\xi x+\eta \omega+\tau t)} K_{1}(x, \omega, t) \mathrm{d} x \mathrm{~d} \omega \mathrm{d} t \\
& =\frac{1}{\sqrt{2 \pi}} \mathcal{F}_{2}\left((x, \omega) \mapsto e^{-\frac{\omega^{2}+x^{2}}{2}} E\left(e^{-i \tau T(1)} \mid X(1)=x, W(1)=\omega\right)\right),
\end{aligned}
$$

where $E\left(e^{-i \tau T(1)} \mid X(1)=x, W(1)=\omega\right)$ expresses the expectation of random variable $T(1)$, recall (20), given the fact that $X(1)=x, W(1)=\omega$. Now by the result of [13] (formula 1.3.4) we have for $d=1$

$$
\begin{aligned}
\left(\mathcal{F}_{3} K_{1}\right)(\xi, \eta, \tau) & =\frac{1}{\sqrt{2 \pi}} \mathcal{F}_{2}\left((x, \omega) \mapsto e^{-\frac{x^{2}+\omega^{2}}{2}} e^{+\frac{x^{2}+\omega^{2}}{2}} \frac{2 \tau}{\sinh (2 \tau)} e^{-|z|^{2} \tau \operatorname{coth}(2 \tau)}\right)(\xi, \eta) \\
& =\frac{1}{(2 \pi)^{\frac{3}{2}}} \frac{1}{\cosh (2 \tau)} e^{-\frac{\xi^{2}+\eta^{2}}{2} \frac{\tanh (2 \tau)}{2 \tau}} .
\end{aligned}
$$

Now since $K_{s}>0$ we have by (18) that $\left\|K_{s}\right\|_{\mathbb{L}_{1}\left(H_{3}\right)}=(2 \pi)^{\frac{3}{2}} \lim _{\tau \rightarrow 0}\left(\mathcal{F}_{3} K_{1}\right)(\mathbf{0}, \tau)=1$. Application of inverse Fourier transform gives

$$
K_{1}(x, \omega, t)=\frac{1}{(2 \pi)^{2}} \int_{\mathbb{R}} \frac{2 \tau}{\sinh (2 \tau)} \cos (\tau t) e^{-\frac{|z|^{2} \tau}{\tanh (2 \tau)}} \mathrm{d} \tau .
$$

\footnotetext{
${ }^{5}$ A Brownian motion is a.e. not differentiable in the classical sense, nor does the integral in (20) make sense in classical integration theory.
} 
Finally identities in (18) provide the general scale space kernel on $H_{3}$ :

$$
K_{s}^{\mathbf{D}}(x, \omega, t)=\frac{1}{(2 \pi s)^{2}} \int_{\mathbb{R}} \frac{2 \tau}{\sinh (2 \tau)} \cos \left(\frac{2 \tau t}{s \sqrt{D_{11} D_{22}}}\right) e^{-\frac{\left(\frac{x^{2}}{D_{11} s}+\frac{\omega^{2}}{D_{22} s}\right) \tau}{2 \tanh (2 \tau)}} \mathrm{d} \tau,
$$

which can be approximated with a one dimensional discrete cosine transform.

The corresponding resolvent kernel $R_{\gamma}(x, \omega, \tau)=\gamma \int_{\mathbb{R}^{+}} K_{s}^{\mathbf{D}}(x, \omega, \tau) e^{-\gamma s} \mathrm{~d} s$ which is again a probability kernel, i.e. $R_{\gamma}>0$ and $\left\|R_{\gamma}\right\|_{\mathbb{L}_{1}\left(H_{3}\right)}=1$, is given by

$$
R_{\gamma}(x, \omega, z)=\frac{2 \gamma \sqrt{\gamma}}{\pi^{2}} \int_{0}^{\infty} \frac{\tau}{\sinh 2 \tau} \operatorname{Re}\left(\frac{k_{1}\left(2 \sqrt{\gamma} \sqrt{\frac{2 \tau}{\tanh 2 \tau}\left(\frac{x^{2}}{D_{11}}+\frac{\omega^{2}}{D_{22}}\right)-\frac{2 i \tau t}{\sqrt{D_{11} D_{22}}}}\right)}{\sqrt{\frac{2 \tau}{\tanh 2 \tau}\left(\frac{x^{2}}{D_{11}}+\frac{\omega^{2}}{D_{22}}\right)-\frac{2 i \tau t}{\sqrt{D_{11} D_{22}}}}}\right) \mathrm{d} \tau
$$

with $k_{1}$ the 1st order BesselK-function.Formulae (22) and (21) are nasty for computation. The resolvent kernel with infinite lifetime is much simpler:

$$
\lim _{\gamma \rightarrow 0} \gamma^{-1} R_{\gamma}(x, \omega, t)=\int_{\mathbb{R}^{+}} K_{s}^{\mathbf{D}}(x, \omega, t) \mathrm{d} s=\frac{1}{2 \pi} \frac{1}{\sqrt{\left(\frac{x^{2}}{D_{11}}+\frac{\omega^{2}}{D_{22}}\right)^{2}+\frac{t^{2}}{D_{11} D_{22}}}},
$$

which follows by taking the limit $\gamma \rightarrow 0$ in (22) and substitution $v=\cosh (2 \tau)$. It provides us the following left invariant metric $d^{\mathbf{D}}: H_{3} \times H_{3} \rightarrow \mathbb{R}^{+}$given by

$$
d^{\mathbf{D}}(g, h)=\sqrt{\left(D_{11}^{-1}\left(x-x^{\prime}\right)^{2}+D_{22}^{-1}\left(\omega-\omega^{\prime}\right)^{2}\right)^{2}+\left(D_{11} D_{22}\right)^{-1}\left(t-t^{\prime}-2\left(x \omega^{\prime}-x^{\prime} \omega\right)^{2}\right)^{2}},
$$

with $g=(x, \omega, t), h=\left(x^{\prime}, \omega^{\prime}, t^{\prime}\right)$. Since (22) is not suitable for practical purposes if $\gamma<\infty$ and since $\tilde{R}_{\gamma=\infty}^{\mathrm{D}}$ decays slowly at infinity we propose to use

$$
\tilde{R}_{\gamma}^{\mathbf{D}}(x, \omega, t)=\frac{4 \gamma}{\pi^{\frac{3}{2}} D_{11} D_{22}} \frac{\exp \left\{-\left(\gamma d^{\mathbf{D}}((x, \omega, t), e)\right)^{2}\right\}}{d^{\mathbf{D}}((x, \omega, t), e)}, \quad e=(0,0,0),
$$

instead. Note that $\lim _{\gamma \rightarrow 0} \gamma \tilde{R}_{\gamma} \equiv \lim _{\gamma \rightarrow 0} \gamma R_{\gamma}, \tilde{R}_{\gamma}>0,\left\|\tilde{R}_{\gamma}\right\|_{\mathbb{L}_{2}\left(H_{3}\right)}=1$ for all $\gamma>0$.

\section{Contour completion and enhancement via left invariant scale spaces on Orientation Scores: $G=\mathrm{SE}(2)$.}

Consider the Euclidean motion group $G=\mathrm{SE}(2)=\mathbb{R}^{2} \rtimes \mathbb{T}$ with group product

$$
\left(g, g^{\prime}\right)=\left(R_{\theta} \mathbf{b}^{\prime}+\mathbf{b}, e^{i\left(\theta+\theta^{\prime}\right)}\right), \quad g=\left(\mathbf{b}, e^{i \theta}\right), g^{\prime}=\left(\mathbf{b}, e^{i \theta^{\prime}}\right) \in G=\mathbb{R}^{2} \rtimes \mathbb{T},
$$

which is (isomorphic to) the group of rotations and translations in $\mathbb{R}^{2}$. Then the tangent space at $e=\left(0,0, e^{i 0}\right)$ is spanned by $\left\{\mathbf{e}_{x}, \mathbf{e}_{y}, \mathbf{e}_{\theta}\right\}=\{(1,0,0),(0,1,0),(0,0,1)\}$ and again by the general recipe (9) we get the following basis for $\mathcal{L}(\mathrm{SE}(2))$ :

$$
\left\{\mathcal{A}_{1}, \mathcal{A}_{2}, \mathcal{A}_{3}\right\}=\left\{\partial_{\theta}, \partial_{\xi}, \partial_{\eta}\right\}=\left\{\partial_{\theta}, \cos \theta \partial_{x}+\sin \theta \partial_{y},-\sin \theta \partial_{x}+\cos \theta \partial_{y}\right\}
$$

with $\xi=x \cos \theta+y \sin \theta, \eta=-x \sin \theta+y \cos \theta$. 
The wavelet transform that maps an image $f \in \mathbb{L}_{2}\left(\mathbb{R}^{2}\right)$ to a so-called orientation score, [4, [5], 8] is given by

$\left(\mathcal{W}_{\psi} f\right)(g)=\left(\mathcal{U}_{g} \psi, f\right)_{\mathbb{L}_{2}\left(\mathbb{R}^{2}\right)}=\int_{\mathbb{R}^{2}} \overline{\psi\left(R_{\theta}^{-1}(\mathbf{y}-\mathbf{x})\right)} f(\mathbf{y}) \mathrm{d} \mathbf{y}, \quad \psi \in \mathbb{L}_{2}\left(\mathbb{R}^{2}\right) \cap \mathbb{L}_{1}\left(\mathbb{R}^{2}\right)$,

where $\psi$ is a suitable line-detecting wavelet in $\mathbb{L}_{2}\left(\mathbb{R}^{2}\right)$ and with representation $\mathcal{U}: \mathrm{SE}(2) \rightarrow \mathcal{B}\left(\mathbb{L}_{2}\left(\mathbb{R}^{2}\right)\right)$ given by

$$
\begin{aligned}
& \mathcal{U}_{g} \psi(\mathbf{y})=\mathcal{T}_{\mathbf{y}} \mathcal{R}_{\theta} \psi(\mathbf{x})=\psi\left(R_{\theta}^{-1}(\mathbf{x}-\mathbf{y})\right), \text { with } R_{\theta}=\left(\begin{array}{c}
\cos \theta-\sin \theta \\
\sin \theta \cos \theta
\end{array}\right) \in S O(2) \leftrightarrow \\
& e^{i \theta} \in \mathbb{T} \text { and } \mathcal{R}_{\theta} \psi(\mathbf{x})=\psi\left(R_{\theta}^{-1} \mathbf{x}\right), \mathcal{T}_{\mathbf{y}} \psi(\mathbf{x})=\psi(\mathbf{x}-\mathbf{y}) .
\end{aligned}
$$

Now $\mathcal{U}$ is reducible and the standard wavelet reconstruction theorems do not apply, nevertheless for proper choice of wavelets one can still obtain quadratic norm preservation. For details, see [8], 4] p.107-146. Now the wavelet transform maps $f$ to a so-called invertible orientation score $U_{f}$, which provides the initial condition $U_{f} \in \mathbb{L}_{2}(\mathrm{SE}(2))$ for our left invariant scale space representations on $S E(2)$ given by (11), generated by (10), which are computed by $G$-convolutions

$W^{\mathbf{D}, \mathbf{a}}(x, y, \theta, s)=\int_{\mathbb{R}^{2}} \int_{0}^{2 \pi} K_{s}^{\mathbf{D}, \mathbf{a}}\left(e^{i\left(\theta-\theta^{\prime}\right)}, R_{\theta^{\prime}}^{-1}\left(\mathbf{x}-\mathbf{x}^{\prime}\right), e^{i\left(\theta-\theta^{\prime}\right)}\right) U_{f}\left(\mathbf{x}^{\prime}, e^{i \theta^{\prime}}\right) \mathrm{d} \theta^{\prime} \mathrm{d} \mathbf{x}^{\prime}$ $P_{\gamma}^{\mathbf{D}, \mathbf{a}}(x, y, \theta)=\int_{\mathbb{R}^{2}} \int_{0}^{2 \pi} R_{\gamma}^{\mathbf{D}, \mathbf{a}}\left(e^{i\left(\theta-\theta^{\prime}\right)}, R_{\theta^{\prime}}^{-1}\left(\mathbf{x}-\mathbf{x}^{\prime}\right), e^{i\left(\theta-\theta^{\prime}\right)}\right) U_{f}\left(\mathbf{x}^{\prime}, e^{i \theta^{\prime}}\right) \mathrm{d} \theta^{\prime} \mathrm{d} \mathbf{x}^{\prime}$.

For explicit formulae of our recently discovered exact convolution kernels $K_{s}^{\mathbf{D}, \mathbf{a}}, R_{\gamma}^{\mathbf{D}, \mathbf{a}} \in \mathbb{L}_{1}(\mathrm{SE}(2))$ we refer to our earlier work [7, with in particular the cases:

1. $Q(\underline{\mathcal{A}})=D_{11}\left(\mathcal{A}_{1}\right)^{2}+\mathcal{A}_{2}$, where the corresponding scale space representation is the Forward Kolmogorov equation of the direction process proposed by Mumford, 14 as a stochastic model for contour completion.

2. $Q(\underline{\mathcal{A}})=D_{11}\left(\mathcal{A}_{1}\right)^{2}+D_{22}\left(\mathcal{A}_{2}\right)^{2}$, where the corresponding scale space representation is the forward Kolmogorov equation of (up to scaling) the stochastic model for perceptual completion (contour enhancement) by Citti et al. 3].

Condition (14) is satisfied, since $\operatorname{span}\left\{\partial_{\theta},\left[\partial_{\theta}, \partial_{\xi}\right],\left[\partial_{\theta},\left[\partial_{\theta}, \partial_{\xi}\right]\right]\right\}=$ span $\left\{\partial_{\theta}, \partial_{\eta},-\partial_{\xi}\right\}=\mathcal{L}(G)$. Although our exact solutions in [7], are simple, they consist of Mathieu-functions with disadvantages concerning computation time. Therefore it is worthwhile to replace the left invariant vector fields of $S E(2)$ (25) by the vector fields $\left\{\hat{A}_{1}, \hat{A}_{2}, \hat{A}_{3}\right\}=\left\{\partial_{\theta}, \partial_{x}+\theta \partial_{y},-\theta \partial_{x}+\partial_{y}\right\}$. This leads to the following approximation 6 in case of contour completion, cf. 4]p.166-167 (for alternatives see [7]):

$$
\begin{aligned}
& \hat{K}_{s}^{D_{11}, a_{1}=1}(x, y, \theta)=\delta(x-t) \frac{\sqrt{3}}{2 D_{11} \pi x^{2}} e^{-\frac{3(x \theta-2 y)^{2}+x^{2}\left(\theta-\kappa_{0} x\right)^{2}}{4 x^{3} D_{11}}} \\
& \hat{R}_{\gamma}^{D_{11}, a_{1}=1}(x, y, \theta)=\alpha \frac{\sqrt{3}}{2 D_{11} \pi x^{2}} e^{-\alpha x} e^{-\frac{3(x \theta-2 y)^{2}+x^{2}\left(\theta-\kappa_{0} x\right)^{2}}{4 x^{3} D_{11}}} \frac{1}{2}(1+\operatorname{sign}(x)) .
\end{aligned}
$$

$\overline{{ }^{6} \text { Note that }}$ the approximation $\hat{K}_{s}^{D_{11}, a_{1}}$, in contrast to the exact solution(s) in 7], has singular behavior because of the violation of (14) as: $\operatorname{span}\left\{\partial_{\theta}\left[\partial_{\theta}, \partial_{x}+\theta \partial_{y}\right],\left[\partial_{\theta},\left[\partial_{\theta}, \partial_{x}+\theta \partial_{y}\right]\right]\right\}=\operatorname{span}\left\{\partial_{\theta}, \partial_{y}\right\}$ which has dimension $2<3$. 
The case of contour enhancement with $\tilde{Q}(\underline{\mathcal{A}})=D_{11}\left(\hat{A}_{1}\right)^{2}+D_{22}\left(\hat{A}_{2}\right)^{2}$ requires a different approach. Here we apply a coordinates transformation

$$
\begin{aligned}
& \hat{K}_{s}^{D_{11}, D_{22}}(x, y, \theta)=\tilde{K}_{s}\left(x^{\prime}, \omega^{\prime}, t^{\prime}\right)=\tilde{K}_{s}\left(\frac{x}{\sqrt{2 D_{11}}}, \frac{\theta}{\sqrt{2 D_{11}}}, \frac{2\left(y-\frac{x \theta}{2}\right)}{\sqrt{D_{11} D_{22}}}\right) \text { where we note } \\
& \partial_{s} \hat{K}_{s}^{D_{11}, D_{22}}=\left(D_{11} \partial_{\theta}^{2}+D_{22}\left(\partial_{x}+\theta \partial_{y}\right)^{2}\right) \hat{K}_{s}^{D_{11}, D_{22}} \Leftrightarrow \\
& \partial_{s} \tilde{K}_{s}^{D_{11}, D_{22}}=\frac{1}{2}\left(\left(\partial_{\omega^{\prime}}-2 x^{\prime} \partial_{t^{\prime}}\right)^{2}+\left(\partial_{x^{\prime}}+2 \omega^{\prime} \partial_{t^{\prime}}\right)^{2}\right) \tilde{K}_{s}^{D_{11}, D_{22}}=\frac{1}{2} \Delta_{K} \tilde{K}_{s}^{D_{11}, D_{22}},
\end{aligned}
$$
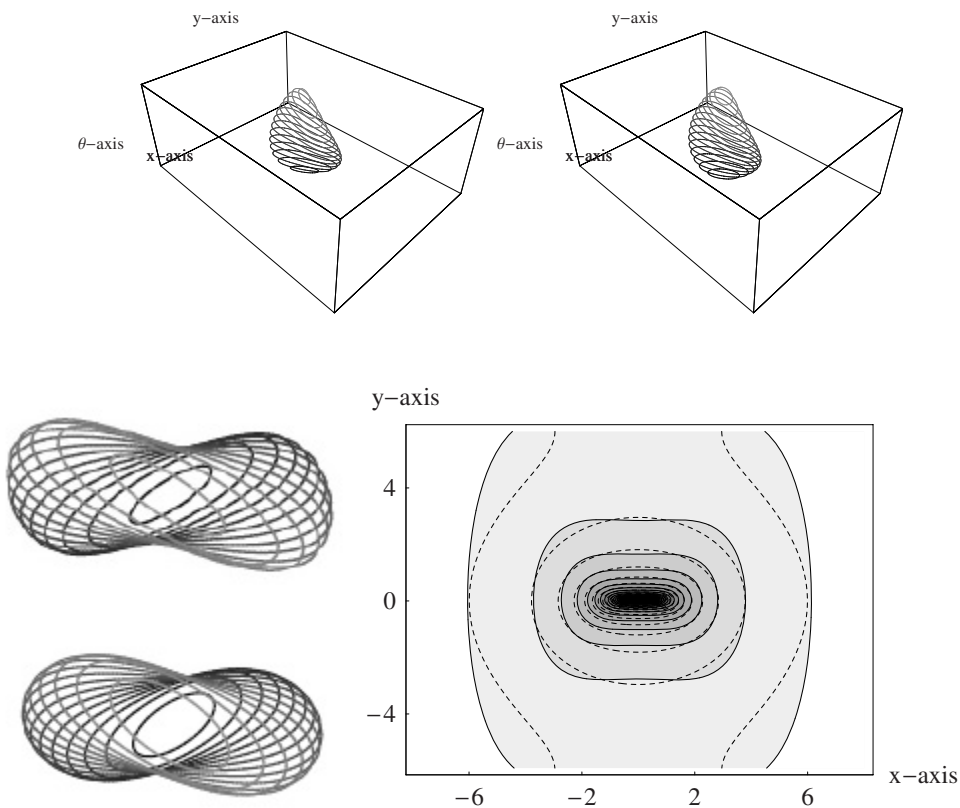

Fig. 2. A comparison between the exact Green's function of the resolvent diffusion process $\left(D_{11} \mathcal{A}_{1}^{2}+D_{22} \mathcal{A}_{2}^{2}-\gamma I\right)^{-1} \delta_{e}, \gamma=\frac{1}{30}, D_{11}=0.1, D_{22}=0.5$ which we explicitly derived in 7] and the approximate Green's function of the resolvent process with infinite lifetime $\lim _{\gamma \rightarrow 0} \gamma^{-1}\left(D_{11} \hat{A}_{1}^{2}+D_{22} \hat{A}_{2}^{2}-\gamma I\right)^{-1} \delta_{e}, D_{11}=0.1, D_{22}=0.5$ given by (27). Top row: a $3 \mathrm{D}$ view on a stack of spacial iso-contours with a $3 \mathrm{D}$-iso-contour of the exact Green's function (right) and the approximate Green's function (left). Bottom-left; a close up on the same stacks of iso-contours but now viewed along the negative $\theta$-axis, with the approximation on top and the exact Green's function below. Bottom-right; an iso-contour-plot of the $x y$-marginal (i.e. Green's function integrated over $\theta$ ) of the exact Green's function with on top the corresponding iso-contours of the approximation in dashed lines. Note that the Green's functions nicely reflect the curvature of the Cartan-connection on $S E(2)$. The stochastic process corresponding to the approximation of the contour enhancement process is given by $X(s)+i \Theta(s)=$ $X(0)+i \Theta(0)+\sqrt{s}\left(\epsilon_{x}+i \epsilon_{\theta}\right)$, where $\epsilon_{x} \sim \mathcal{N}\left(0,2 D_{11}\right), \epsilon_{\theta} \sim \mathcal{N}\left(0,2 D_{22}\right)$ and, by (20), $Y(s)=\frac{X(s) \Theta(s)}{2}+\frac{1}{2} \int_{0}^{s} \Theta \mathrm{d} X-X \mathrm{~d} \Theta=\int_{0}^{s} \Theta(t)-\Theta(0) \mathrm{d} t$. 
which is exactly the evolution equation on $H_{3}$ generated by Kohn's Laplacian considered in the previous example ! As a result we have 7

$$
\begin{aligned}
& \hat{K}_{s}^{D_{11}, D_{22}}(x, y, \theta)=\frac{1}{2 D_{11} D_{22}} \tilde{K}_{s}^{H_{3}}\left(\frac{x}{\sqrt{2 D_{11}}}, \frac{\theta}{\sqrt{2 D_{11}}}, \frac{2\left(y-\frac{x \theta}{2}\right)}{\sqrt{D_{11} D_{22}}}\right) \\
& \quad=\frac{1}{8 D_{11} D_{22} \pi^{2} s^{2}} \int_{\mathbb{R}} \frac{2 \tau}{\sinh (2 \tau)} \cos \left(\frac{2 \tau\left(y-\frac{x \theta}{2}\right)}{s \sqrt{D_{11} D_{22}}}\right) e^{-\frac{\left(\frac{x^{2}}{s D_{22}}+\frac{\theta^{2}}{s D_{11}}\right) \tau}{\tanh (2 \tau)}} \mathrm{d} \tau \\
& \lim _{\gamma \rightarrow \infty} \gamma^{-1} \hat{R}_{\gamma}(x, y, \theta)=\frac{1}{4 \pi D_{11} D_{22}} \frac{1}{\sqrt{\frac{1}{16}\left(\frac{x^{2}}{D_{22}}+\frac{\theta^{2}}{D_{11}}\right)^{2}+\frac{\left(y-\frac{1}{2} x \theta\right)^{2}}{D_{11} D_{22}}}} .
\end{aligned}
$$

See Figure 2 ,

\section{Conclusion}

We derived a unifying framework for scale spaces (related to stochastic processes) on Lie-groups. These scale spaces are directly linked to operators on images by means of unitary wavelet transforms. To obtain proper invariance of these operators, the scale spaces must be left-invariant and thereby its solutions are $G$-convolutions with Green's functions. As this framework lead to fruitful applications on contour completion, contour enhancement and adaptive non-linear diffusion, see [9], 8], [5], in the special case $G=S E(2)$, this theory can be further employed for other groups, such as the Heisenberg group, $G=H_{2 d+1}$.

\section{References}

1. S.T. Ali, J.P. Antoine, and J.P. Gazeau. Coherent States, Wavelets and Their Generalizations. Springer Verlag, New York, Berlin, Heidelberg, 1999.

2. A.V. Bukhvalov and Arendt W. Integral representation of resolvent and semigroups. Forum Math. 6, 6(1):111-137, 1994.

3. G. Citti and A. Sarti. A cortical based model of perceptual completion in the roto-translation space. pages 1-27, 2004. Pre-print, available on the web http://amsacta.cib.unibo.it/archive/00000822.

4. R. Duits. Perceptual Organization in Image Analysis. $\mathrm{PhD}$ thesis, Eindhoven University of Technology, Dep. of Biomedical Engineering, The Netherlands, 2005.

5. R. Duits, M. Felsberg, G. Granlund, and B.M. ter Haar Romeny. Image analysis and reconstruction using a wavelet transform constructed from a reducible representation of the euclidean motion group. IJCV. Accepted for publication. To appear in 2007 Volume 72 issue 1, p.79-102.

6. R. Duits, L.M.J. Florack, J. de Graaf, and B. ter Haar Romeny. On the axioms of scale space theory. Journal of Math. Imaging and Vision, 20:267-298, 2004.

7. R. Duits and M.A. van Almsick. The explicit solutions of linear left-invariant second order stochastic evolution equations on the $2 \mathrm{~d}$-euclidean motion group. Accepted for publication in Quarterly of Applied Mathematics, AMS, 2007.

8. R. Duits and M.A. van Almsick. Invertible orientation scores as an application of generalized wavelet theory. Image Processing, Analysis, Recognition and Understanding, 17(1):42-75, 2007.

\footnotetext{
7 Note that our approximation of the Green's function on the Euclidean motion group does not coincide with the formula by Citti in 3 .
} 
9. E. Franken, R. Duits, and B.M. ter Haar Romeny. Non-linear diffusion on the euclidean motion group. Accepted for Publication in the Proceedings of SSVM-07, conf. on Scale Space and Var. Methods in Computer Vision., 2006.

10. B. Gaveau. Principe de moindre action, propagation de la chaleur et estimees sous elliptiques sur certains groupes nilpotents. Acta mathematica, 139:96-153, 1977.

11. W. Hebisch. Estimates on the semigroups generated by left invariant operators on lie groups. Journal fuer die reine und angewandte Mathematik, 423:1-45, 1992.

12. L. Hormander. Hypoellptic second order differential equations. Acta Mathematica, 119:147-171, 1968.

13. P. Lévy. Wiener random functions and other laplacian random functions. In Proc. of the 2nd Berkely Symposium, pages 171-187, USA, 1950. California Press.

14. D. Mumford. Elastica and computer vision. Algebraic Geometry and Its Applications. Springer-Verlag, pages 491-506, 1994.

15. C. Sagiv, N. A. Sochen, and Y. Y. Zeevi. Scale space generation via uncertainty principles. In Scale Space and PDE Methods in Computer Vision 2005, pages 351-362. Springer-Verlag. 